\title{
Bumpless transfer for discrete-time switched systems
}

\author{
I. Malloci, L. Hetel, J. DaAfouz, Member, IEEE, C. Iung, Member, IEEE, and R. Bonidal
}

\begin{abstract}
A bumpless transfer method for discrete-time switched linear systems is presented. It is based on an additional controller which is activated at the switching time for reducing the control discontinuities. Dwell time conditions to guarantee the stability of the closed-loop system are provided. Simulation tests on the Eisenhüttenstadt hot strip mill of ArcelorMittal are shown.
\end{abstract}

\section{INTRODUCTION}

In practical control problems, several linear controllers are often used to control the same non-linear plant, one for each operating point. This strategy avoids the non-linear control design, certainly more complicated. Nevertheless, switching among more controllers implies control discontinuities and undesired transient behaviors. Moreover, industrial systems are often characterized by saturations due to the actuators limits and do not accept these discontinuities. Consequently, the dynamics performance can be largely modified and the stability may not be guaranteed anymore. Slope saturations of the control signal are well-known for their destabilizing effect. The solution of this problem is called bumpless transfer.

A description of most popular strategies for the bumpless transfer problem can been found in [8], [9], [10] and [13]. One of the first bumpless transfer schemes is proposed by [11] for non-linear plants. The idea consists in pre-setting the off-line controller state in order to reduce the transient behavior at the switching time. In [18] and [19], results of [11] are generalized for controllers which are not biproper and have a minimum phase. A linear quadratic (LQ) optimization method is introduced to minimize the distance between the on-line and the off-line controller output. In [5], the discontinuity of the controller output is reduced resetting the fast dynamics of the controller at the switching time. In [21], the desired transient behavior is obtained using the $\mathcal{L}_{2}$ anti-windup structure [17].

Although the bumpless transfer problem has been widely studied in literature, only few articles address the switched systems framework. In [1], a bumpless transfer solution for continuous-time switched systems where the order of the controller is smaller than the order of the plant is given. The idea consists in forcing the output of the activated controller

This work has been supported by grants from "la région Lorraine, France" and ArcelorMittal Maizières Research.

I. Malloci, L. Hetel, J. Daafouz and C. Iung are with the Centre de Recherche en Automatique de Nancy, UMR 7039 CNRS - Nancy Université, ENSEM, 2, Avenue de la forêt de Haye 54516 Vandoeuvre-lès-Nancy, France. Corresponding author: Jamal.Daafouz@ensem.inpl-nancy.fr.

R. Bonidal is with ArcelorMittal Maizières, R\&D Industrial Operations, BP 30320, F-57283 Maizières-lès-Metz Cedex, France. to be equal to the plant input at the switching time. An analogous strategy is proposed in [7] for continuous-time LPV systems. However, as pointed out in [20] and [21], a constraint on the controller output does not guarantee better performances of the plant output.

In this article, a bumpless transfer control design for discrete-time switched systems is presented. We propose an additional controller which is activated at each switching time. The controller and the plant output are forced to follow a desired profile for a given period of time. This minimizes the control discontinuity guaranteeing the plant output performances. The solution is based on the LQ optimization theory, that has been introduced on the bumpless transfer framework by [19]. Stability of the closed-loop system is guaranteed by LMI conditions [2] using multiple Lyapunov functions [3] and the dwell time approach [15].

The article is organized as follows. In the next section, the problem is formulated. In section III, the optimization criterion to design the bumpless transfer controller (BT controller) is detailed. In section IV, stability conditions for the closed-loop system are investigated. In section V, simulation results of the Eisenhüttenstadt hot strip mill (Germany) are presented. Finally, a conclusion is given.

\section{PROBLEM FORMULATION}

Consider the discrete-time switched system

$$
\left\{\begin{array}{l}
x_{k+1}=A_{\sigma(k)} x_{k}+B_{\sigma(k)} u_{k} \\
y_{k}=C_{\sigma(k)} x_{k}
\end{array}\right.
$$

where $x \in \mathbb{R}^{n}$ is the state, $u \in \mathbb{R}^{r}$ is the control signal, $y \in \mathbb{R}^{m}$ is the output signal and $\sigma(k): \mathbb{N} \rightarrow \Gamma=\{1, \ldots, N\}$ is the switching signal, which is assumed to be unknown a priori but available in real time. The minimal interval of time between two switchings $D_{j}$ is assumed to be known. Moreover, the pair $\left(A_{j}, B_{j}\right)$ is supposed to be controllable and the pair $\left(A_{j}, C_{j}\right)$ observable, $\forall j \in \Gamma$. Furthermore, the state $x$ is supposed to be available for feedback and the plant matrices (1) are assumed to be well-known. A state-feedback control law

$$
u_{k}=K_{\sigma(k)} x_{k}
$$

which stabilizes the closed-loop system (1)-(2) is given. In order to reduce the amplitude of the control signal discontinuities, different strategies are possible. Here, a BT controller which is activated at the switching time for the 
period of time $\tau_{j}^{M}<D_{j}$ is proposed, that is:

$$
u_{k}=\left\{\begin{array}{lll}
K_{j} x_{k}+u_{k}^{b t} & \text { if } & \tau_{j} \leq \tau_{j}^{M} \\
K_{j} x_{k} & \text { if } & \tau_{j}>\tau_{j}^{M}
\end{array}\right.
$$

where $u^{b t} \in \mathbb{R}^{r}$ is the BT controller output and $\tau_{j} \in \Xi=$ $\left\{1, \ldots, \tau_{j}^{M}\right\}$ the number of times between the present time $k$ and the last switching. For each mode $j \in \Gamma$, the closed-loop system (Fig. 1) can be written as

$$
\left\{\begin{array}{l}
x_{k+1}=\left(A_{j}+B_{j} K_{j}\right) x_{k}+B_{j} u_{k}^{b t} \\
y_{k}=C_{j} x_{k} .
\end{array}\right.
$$

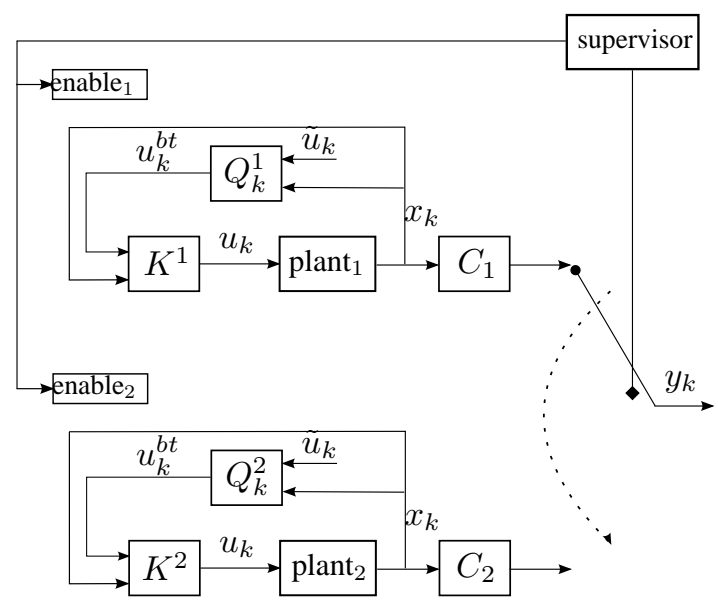

Fig. 1. Closed-loop system with $\Gamma=\{1,2\}$

The BT controller $Q^{j}$ is designed in the next section. The signal $u_{k}-\tilde{u}_{k}^{j}$ is minimized using a LQ criterion, where $\tilde{u}^{j}$ represents the desired profile of the control signal. For simplicity reasons, a straight line is chosen as desired profile. Let $t_{j}$ be the switching time from the subsystem $i$ to the subsystem $j, \forall(i, j) \in \Gamma \times \Gamma$. We can define

$$
\tilde{u}_{\tau_{j}}^{j}=\tilde{u}_{j}^{0}+p_{j} \tau_{j}
$$

where

$$
\tilde{u}_{j}^{0}=K_{i} x_{t_{j}-1}
$$

is the control signal value at the time before the switching and $p_{j}$ determines the slope of the desired profile, i. e.

$$
p_{j}=\frac{1}{\tau_{j}^{M}}\left(K_{j} x_{t_{j}}-K_{i} x_{t_{j}-1}\right) .
$$

We obtain a value of $p$ which depends on the control signal discontinuity (Fig. 2). In order to guarantee the performances of the plant output $y$, also the difference between $y$ and the desired plant output $\tilde{y}$ is minimized.

\section{BUMPLESS TRANSFER CONTROLLER DESIGN}

In this section, the method to design the BT controller is presented. In order to follow the desired profiles $\tilde{u}$ and $\tilde{y}$, the problem is formulated as a classical LQ optimization problem. In the bumpless transfer framework, this solution

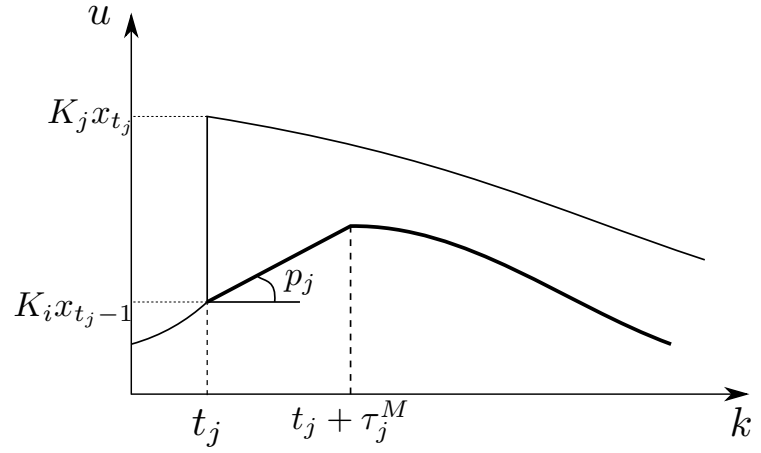

Fig. 2. $u$ evolution with BT controller switched on (thick line) and switched off (thin line)

has been proposed by [19], where the difference between the on-line and the off-line controller output is minimized before each switching. Moreover, the difference between the on-line and the off-line controller input is minimized. This strategy initializes the controller state and then reduces the transient behavior on the plant output. Since the antibumpless action consists in pre-setting the state of the offline controller before the switching, the method does not address control systems without memory, such as statefeedback control laws. Another problem concerns the stability of the closed-loop system, which is not guaranteed when arbitrary switchings occur. To solve these problems, for discrete-time switched systems we propose a BT controller which is activated at each switching time. Stability conditions for the closed-loop system are given in the next section. For each mode $j \in \Gamma$, the design of the BT controller is based on the minimization of the following quadratic cost function:

$$
J_{j}=\phi_{T_{f}^{j}}^{j}+\frac{1}{2} \sum_{k=0}^{T_{f}^{j}-1}\left[z_{k}^{u^{\prime}} W_{j}^{u} z_{k}^{u}+z_{k}^{y^{\prime}} W_{j}^{y} z_{k}^{y}\right],
$$

with

$$
\begin{gathered}
z_{k}^{u}=u_{k}-\tilde{u}_{k}^{j} \\
z_{k}^{y}=y_{k}-\tilde{y}_{k}^{j} \\
\phi_{T_{f}^{j}}^{j}=\frac{1}{2} z_{T_{f}^{j}}^{u^{\prime}} P^{j} z_{T_{f}^{j}}^{u}
\end{gathered}
$$

where $W_{j}^{u}$ and $W_{j}^{y}$ are positive definite weighting matrices of appropriated dimensions. $T_{f}^{j}=\tau_{j}^{M}+1$ is the terminal time and $P^{j}$ is a positive semi-definite terminal weighting matrix. For simplicity reasons, we consider $\tilde{y}^{j}=0$. The next theorem shows how to compute the signal $u^{b t}$. The proof, which is based on the Pontryagin's minimum principle [4], is similar to the one given in [19].

Theorem 1: Given the system (4), the quadratic cost function (8) and the terminal time $T_{f}^{j}$, the BT controller which minimizes the signals $z^{u}$ and $z^{y}$ is given by

$$
u_{k}^{b t}=Q_{k}^{j}\left[\begin{array}{c}
x_{k} \\
\tilde{u}_{k}^{j} \\
g_{k+1}^{j}
\end{array}\right]
$$


with

$$
Q_{k}^{j}=\left[\begin{array}{c}
\left(\tilde{N}_{k+1}^{j} \Pi_{k+1}^{j} A_{j}-K_{j}\right)^{\prime} \\
\left(I+\tilde{N}_{k+1}^{j} \Pi_{k+1}^{j} B_{j}\right)^{\prime} \\
-\tilde{N}_{k+1}^{j^{\prime}}
\end{array}\right]^{\prime}
$$

and

$$
\tilde{N}_{k+1}^{j}=-\left(W_{j}^{u}\right)^{-1} B_{j}^{\prime}\left(I-\Pi_{k+1}^{j} \tilde{B}_{j}\right)^{-1},
$$

$\forall j \in \Gamma$. The values of $\Pi^{j}$ and $g^{j}$ are given by the equations

$$
\Pi_{k}^{j}=A_{j}^{\prime}\left(I-\Pi_{k+1}^{j} \tilde{B}_{j}\right)^{-1} \Pi_{k+1}^{j} A_{j}+\tilde{C}_{j}
$$

and

$$
g_{k}^{j}=A_{j}^{\prime}\left(I-\Pi_{k+1}^{j} \tilde{B}_{j}\right)^{-1}\left(g_{k+1}^{j}-\Pi_{k+1}^{j} B_{j} \tilde{u}_{k}^{j}\right)
$$

with

$$
\begin{aligned}
& \tilde{B}_{j}=-B_{j}\left(W_{j}^{u}\right)^{-1} B_{j}^{\prime} \\
& \tilde{C}_{j}=C_{j}^{\prime} W_{j}^{y} C_{j} .
\end{aligned}
$$

The bound condition is

$$
\begin{aligned}
& \Pi_{T_{f}^{j}}^{j}=0 \\
& g_{T_{f}^{j}}^{j}=0 .
\end{aligned}
$$

Remark 1: In the finite horizon approach, the knowledge of all the future values of $\tilde{u}$ is required in order to solve (15) backward in time. Then, in general this method cannot be applied to solve practical problems, as discussed in [19]. Nevertheless, from (5), in our case all the values of $\tilde{u}$ can be computed in the finite horizon $\Xi$. Only the knowledge of $x_{t_{j}-1}$ and $x_{t_{j}}$ is needed. Since these informations are available at each switching time, the method can always be used.

\section{STABILITY ANALYSIS}

In the previous sections, we assumed that the BT controller is switched on for $\tau_{j}^{M}$ times. As the original controller (2) has been designed without taking into account this fact, the stability is not guaranteed anymore. Then, in this section, a stability condition for the closed-loop system (4) is given. For each mode $j \in \Gamma$, the closed-loop system

$$
x_{k+1}=\left(A_{j}+B_{j} K_{j}\right) x_{k}+B_{j} u_{k}^{b t}
$$

can be written in the equivalent form

$$
v_{k+1}=Y_{j}\left(\tau_{j}\right) v_{k}
$$

where

$$
v_{k}=\left[\begin{array}{c}
x_{k} \\
x_{k-1} \\
\tilde{u}_{k}^{0} \\
p_{k}
\end{array}\right]
$$

is the augmented state and the signals $\tilde{u}^{0}$ and $p$ are defined in section II. The representation of a switched system with an augmented state approach is justified in [12]. We distinguish two phases on the interval between two switchings:
- The bumpless transfer phase: the BT controller is on. We find

$$
\left.Y_{j}\left(\tau_{j}\right)\right|_{\tau_{j}=1}=\left[\begin{array}{cccc}
\bar{H}_{\tau_{j}+1}^{j} & \bar{L}_{\tau_{j}+1}^{j} & 0 & 0 \\
I & 0 & 0 & 0 \\
0 & K_{i} & 0 & 0 \\
\frac{K_{j}}{\tau_{j}^{M}} & -\frac{K_{i}}{\tau_{j}^{M}} & 0 & 0
\end{array}\right]
$$

and

$$
\left.Y_{j}\left(\tau_{j}\right)\right|_{2 \leq \tau_{j} \leq \tau_{j}^{M}}=\left[\begin{array}{cccc}
\bar{A}_{\tau_{j}+1}^{j} & 0 & \bar{U}_{\tau_{j}+1}^{j} & \bar{P}_{\tau_{j}+1}^{j} \\
I & 0 & 0 & 0 \\
0 & 0 & I & 0 \\
0 & 0 & 0 & I
\end{array}\right] .
$$

In this case, the stability of (18) is not guaranteed. The construction of $Y_{j}$ is detailed in the appendix I.

- The recuperation phase: the $\mathrm{BT}$ controller is off. We have

$$
\left.Y_{j}\left(\tau_{j}\right)\right|_{\tau_{j}>\tau_{j}^{M}}=Y_{j}^{s}=\left[\begin{array}{cccc}
A_{j}+B_{j} K_{j} & 0 & \cdots & 0 \\
I & 0 & \cdots & 0 \\
0 & \cdots & & 0 \\
\vdots & & & \vdots \\
0 & \cdots & & 0 .
\end{array}\right],
$$

where $Y_{j}^{s}$ is Schur and constant $\forall j \in \Gamma$.

Given the minimal interval of time between two switchings $D_{j}$, the value of $\tau_{j}^{M}$ can always be reduced in order to make the system (18) stable. Let define the transition matrix between two switching times

$$
Z_{j}\left(D_{j}, \tau_{j}^{M}\right)=\left.\prod_{\tau_{j}=1}^{\tau_{j}^{M}} Y_{j}\left(\tau_{j}\right)\right|_{\tau_{j} \leq \tau_{j}^{M}} Y_{j}^{s\left(D_{j}-\tau_{j}^{M}-1\right)} .
$$

The following theorem checks the closed-loop system stability.

Theorem 2: Given $D_{j}$, if there exist positive definite matrices $P_{Y_{j}}=P_{Y_{j}}^{\prime}, P_{Z_{j}}=P_{Z_{j}}^{\prime}$ of appropriate dimensions and scalars $\tau_{j}^{M}$ such that the LMIs

$$
\begin{aligned}
Y_{j}^{s^{\prime}} P_{Y_{j}} Y_{j}^{s}-P_{Y_{j}} & \prec 0 \\
Z_{j}^{\prime} P_{Z_{j}} Z_{j}-P_{Z_{j}} & \prec 0 \\
Z_{j}^{\prime} P_{Y_{j}} Z_{j}-P_{Z_{j}} & \prec 0 \\
Y_{j}^{s^{\prime}} P_{Z_{i}} Y_{j}^{s}-P_{Y_{j}} & \prec 0
\end{aligned}
$$

are verified $\forall(i, j) \in \Gamma \times \Gamma$, then the closed-loop system (18) is asymptotically stable.

We give an idea of the proof. In the case of arbitrary switching law, a necessary condition for the asymptotic stability of the closed-loop system (18) is that each subsystem is stable. Since for assumption $Y^{s}$ is Schur, from (19) there exists a value of $\tau_{j}^{M}$ such that also the subsystem $Z_{j}$ is Schur, $\forall j \in \Gamma$. In Fig. 3, the system evolution is shown for $\Gamma=\{1,2\}$. For the closed-loop system stability, 


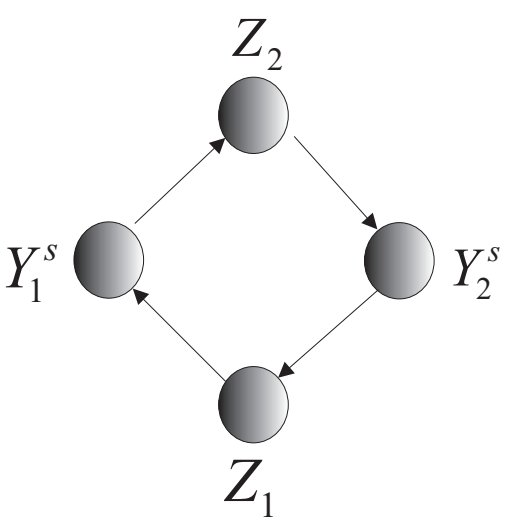

Fig. 3. System evolution

TABLE I

BT CONTROLLER DATA

\begin{tabular}{|c||c|c|c|}
\hline & 2 & 3 & 4 \\
\hline \hline$D_{j}$ & 64 & 38 & 30 \\
\hline$\tau_{j}^{M}$ & 30 & 15 & 6 \\
\hline$W_{j}^{u}$ & $I$ & $100 I$ & $10 I$ \\
\hline$W_{j}^{y}$ & $I$ & $I$ & $I$ \\
\hline
\end{tabular}

we also must check that switching among the subsystems leads to a stable behavior. This is the meaning of the LMIs (22) and (23). Thus, the conditions of Theorem 2 guarantee a decreasing trajectory after switching. This is equivalent to say that the closed-loop system (18) is stable [16].

\section{SIMULATION RESULTS}

In this section, the strategy proposed in the previous sections is applied to the Eisenhüttenstadt hot strip mill (HSM) of ArcelorMittal. The rolling process consists in crushing a metal strip between two rolls in inverse rotation for obtaining a strip with constant and desired thickness. A HSM is the association of several stands in a line, where each stand is constituted by a set of rolls. The lateral movement of the strip, with reference to the mill axis, may induce a decrease of the product quality and rolls damage. Then, in order to improve the reliability and the process quality, this displacement must be reduced [6]. At the end of the treatment, the strip leaves the stands one after the others. Each time the strip leaves a stand, the system dynamics changes. The HSM is modeled as a switched system with four subsystems and three switchings [14]. For each subsystem $j \in \Gamma$, a different discrete-time state-feedback control gain $K_{j}$ has been designed. Given the weighting matrices $W^{u}, W^{y}$ and the minimal dwell time $D_{j}$ for a product of the HSM database, the choice of the $\tau_{j}^{M}$ values summarized in Table I allow to find a solution for the LMI conditions of Theorem 2. This guarantees the stability of the closedloop system. $I$ denotes an identity matrix of appropriate dimension. Since the system never switches back to the first
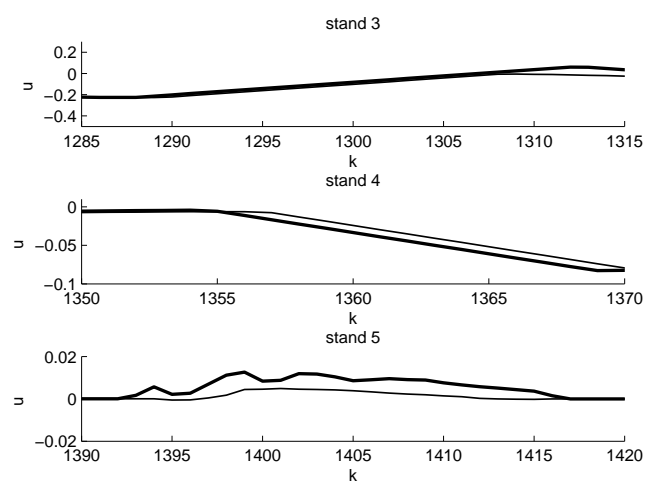

Fig. 4. $u$
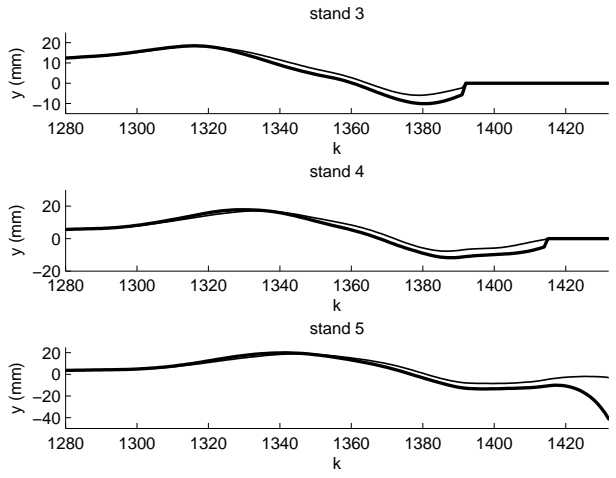

Fig. 5. $y$

subsystem, no BT controller is designed for $j=1$.

The signal $u^{b t}$ is computed applying Theorem 1. Equations (13) and (14) can be solved off-line. At the opposite, to compute the equation (15) we need to know the $x_{t_{j}-1}$ value, where $t_{j}$ is the switching time to the subsystem $j$. Then, it can be computed only on-line, at the switching time. Since the output signal $y$ corresponds to the displacement that has to be minimized, we choose $\tilde{y}=0$. Each stand is controlled by a different control signal. For the simulated product, switchings occur at the instants $k=1290, k=1354$ and $k=1392$. In Fig. 4 it is shown the controller output $u$ for the last three stands. For each stand, we propose a zoom of the zone corresponding to the biggest bumps on $u$. The bold line shows the $u$ evolution without $\mathrm{BT}$ control whereas the thinnest line shows the $u$ evolution when the BT controller is on. The evolution of the strip displacement $y$ is shown in Fig. 5. As expected, performances are better when the BT controller is on (thinnest line). In particular, the strip displacement in the exit of the system (stand 5 in Fig. 5) is reduced from 40 to $8 \mathrm{~mm}$.

\section{CONCLUSION}

In this article, a bumpless transfer method for discretetime switched systems has been proposed. The BT controller has been designed using a LQ optimization method. The idea consists in forcing the controller output and the plant 
output to follow a desired profile. This allows to avoid the control signal discontinuities and to improve the system performances. A LMI criterion guaranteeing the stability of the closed-loop system is proposed. Simulation tests on the Eisenhüttenstadt HSM of ArcelorMittal are provided.

\section{APPENDIX I}

\section{CONSTRUCTION OF $Y_{j}$}

Consider the switching from the subsystem $i$ to the subsystem $j$. From Theorem 1, when the BT controller is on

$$
u_{k}^{b t}=Q_{\tau_{j}}^{j}\left[\begin{array}{c}
x_{k} \\
\tilde{u}_{\tau_{j}}^{j} \\
g_{\tau_{j}+1}^{j}
\end{array}\right]
$$

where

$$
\tilde{u}_{\tau_{j}}^{j}=\tilde{u}_{j}^{0}+p_{j} \tau_{j}
$$

with

$$
\tilde{u}_{j}^{0}=K_{i} x_{t_{j}-1}
$$

and

$$
p_{j}=\frac{1}{\tau_{j}^{M}}\left(K_{j} x_{t_{j}}-K_{i} x_{t_{j}-1}\right) .
$$

The evolution of the signal $g^{j}$ in (24) is given by (15), which can be rewritten as

$$
g_{\tau_{j}}^{j}=G_{\tau_{j}}^{j, u} \tilde{u}_{j}^{0}+G_{\tau_{j}}^{j, p} p_{j}
$$

where

$$
\begin{gathered}
G_{\tau_{j}}^{j, u}=-\sum_{\eta=0}^{\tau_{j}^{M}-\tau_{j}}\left(\prod_{\zeta=\tau_{j}+1}^{\tau_{j}^{M}+1-\eta} M_{\zeta}^{j}\right) \Pi_{\tau_{j}+1-\eta}^{j} B^{j}, \\
G_{\tau_{j}}^{j, p}=-\sum_{\eta=0}^{\tau_{j}^{M}-\tau_{j}}\left(\prod_{\zeta=\tau_{j}+1}^{\tau_{j}^{M}+1-\eta} M_{\zeta}^{j}\right) \Pi_{\tau_{j}+1-\eta}^{j} B^{j}\left(\tau^{M}-\eta\right)
\end{gathered}
$$

and

$$
M_{\tau_{j}}^{j}=A_{j}^{\prime}\left(I-\Pi_{\tau_{j}}^{j} \tilde{B}_{j}\right)^{-1} .
$$

The closed form (28) allows to express (15) as a function of $\tilde{u}_{j}^{0}$ and $p_{j}$. Then, the system (17) becomes

$$
\begin{aligned}
x_{k+1}= & \bar{A}_{\tau_{j}+1}^{j} x_{k}+\left(\bar{B}_{\tau_{j}+1}^{j}-B_{j} \tilde{N}_{\tau_{j}+1}^{j} G_{\tau_{j}+1}^{j, u}\right) \tilde{u}_{j}^{0}+ \\
& \left(\tau_{j} \bar{B}_{\tau_{j}+1}^{j}-B_{j} \tilde{N}_{\tau_{j}+1}^{j} G_{\tau_{j}+1}^{j, p}\right) p_{j}
\end{aligned}
$$

with

$$
\bar{A}_{\tau_{j}}^{j}=\left(I+B_{j} \tilde{N}_{\tau_{j}}^{j} \Pi_{\tau_{j}}^{j}\right) A_{j}
$$

and

$$
\bar{B}_{\tau_{j}}^{j}=B_{j}\left(I+\tilde{N}_{\tau_{j}}^{j} \Pi_{\tau_{j}}^{j} B_{j}\right) .
$$

When $\tau_{j}=1, t_{j}=k$. Then $\tilde{u}_{j}^{0}$ and $p_{j}$ can be initialized as function of $x_{k}$ and $x_{k-1}$. Using (26) and (27), we find

$$
\left.Y_{j}\left(\tau_{j}\right)\right|_{\tau_{j}=1}=\left[\begin{array}{cccc}
\bar{H}_{\tau_{j}+1}^{j} & \bar{L}_{\tau_{j}+1}^{j} & 0 & 0 \\
I & 0 & 0 & 0 \\
0 & K_{i} & 0 & 0 \\
\frac{K_{j}}{\tau_{j}^{M}} & -\frac{K_{i}}{\tau_{j}^{M}} & 0 & 0
\end{array}\right]
$$

with

$$
\bar{H}_{\tau_{j}}^{j}=\bar{A}_{\tau_{j}}^{j}+\frac{1}{\tau_{j}^{M}}\left(\bar{B}_{\tau_{j}}^{j}-B_{j} \tilde{N}_{\tau_{j}}^{j} G_{\tau_{j}}^{j, p}\right) K_{j}
$$

and

$\bar{L}_{\tau_{j}}^{j}=\left(\bar{B}_{\tau_{j}}^{j}-B_{j} \tilde{N}_{\tau_{j}}^{j} G_{\tau_{j}}^{j, u}\right) K_{i}-\frac{1}{\tau_{j}^{M}}\left(\bar{B}_{\tau_{j}}^{j}-B_{j} \tilde{N}_{\tau_{j}}^{j} G_{\tau_{j}}^{j, p}\right) K_{i}$.

When $2 \leq \tau_{j} \leq \tau_{j}^{M}, \tilde{u}_{j}^{0}$ and $p_{j}$ remains constant. We have

$$
\left.Y_{j}\left(\tau_{j}\right)\right|_{2 \leq \tau_{j} \leq \tau_{j}^{M}}=\left[\begin{array}{cccc}
\bar{A}_{\tau_{j}+1}^{j} & 0 & \bar{U}_{\tau_{j}+1}^{j} & \bar{P}_{\tau_{j}+1}^{j} \\
I & 0 & 0 & 0 \\
0 & 0 & I & 0 \\
0 & 0 & 0 & I
\end{array}\right]
$$

with

$$
\bar{U}_{\tau_{j}}^{j}=\bar{B}_{\tau_{j}}^{j}-B_{j} \tilde{N}_{\tau_{j}}^{j} G_{\tau_{j}}^{j, u}
$$

and

$$
\bar{P}_{\tau_{j}}^{j}=\tau_{j} \bar{B}_{\tau_{j}}^{j}-B_{j} \tilde{N}_{\tau_{j}}^{j} G_{\tau_{j}}^{j, p} .
$$

When the BT controller is off $u_{k}^{b t}=0$, then

$$
\left.Y_{j}\left(\tau_{j}\right)\right|_{\tau_{j}>\tau_{j}^{M}}=Y_{j}^{s}=\left[\begin{array}{cccc}
A_{j}+B_{j} K_{j} & 0 & \cdots & 0 \\
I & 0 & \cdots & 0 \\
0 & \cdots & & 0 \\
\vdots & & & \vdots \\
0 & \cdots & & 0
\end{array}\right]
$$

is constant $\forall j \in \Gamma$.

\section{REFERENCES}

[1] A.B. Arehart and W.A. Wolovich. Bumpless switching controllers. In Conference on Decision and Control, 1996.

[2] S. Boyd, L.E. Ghaoui, E. Feron, and V. Balakrishnan. Linear Matrix Inequalities in system and control theory. Society for Industrial and Applied Mathematics, 1994.

[3] S. Branicky. Multiple Lyapunov function andother analysis tools for switched and hybrid systems. IEEE Transactions on Automatic Control, 43(4):475-482, 1998.

[4] A. E. Bryson and Y. C. Ho. Applied optimal control. John Wiley, 1975.

[5] S.Y. Cheong and M.G. Safonov. Bumpless transfer for adaptive switching controls. In IFAC World Congress, 2008.

[6] J. Daafouz, R. Bonidal, C. Iung, P. Szczepanski, N. Naumann, and U. Koschack. New steering control at EKO Stahl finishing mill. In The Iron \& Steel Technology Conference and Exposition, 2008.

[7] K. Dong and F. Wu. Online switching control design of LFT systems. In Conference on Decision and Control, 2006.

[8] C. Edwards and I. Postlethwaite. Anti-windup bumpless transfer schemes. Automatica, 34(2):199-210, 1998.

[9] S.F. Graebe and A. Ahlen. The control handbook. CRC press, 1996.

[10] R. Hanus. Anti-windup and bumpless transfer: A survey. In 12th IMACS world congress, 1988.

[11] R. Hanus, M. Kinnaert, and J. Henrotte. Conditioning technique, a general anti-windup and bumpless transfer method. Automatica, 23(6):729-739, 1987.

[12] L. Hetel, J. Daafouz, and C. Iung. Equivalence between the LyapunvKrasovksii functional approach for discrete delay systems and the stability conditions for switched systems. In IFAC workshop on Time Delay Systems, 2007.

[13] M.V. Kothare, P.J. Campo, M. Morari, and N. Nett. A unified framework for study of anti-windup designs. Automatica, 30(12):18691883, 1994.

[14] I. Malloci, J. Daafouz, C. Iung, R. Bonidal, and P. Szczepanski. Switched system modeling and robust steering control of the tail end phase in a hot strip mill. To appear in Nonlinear Analysis: Hybrid Systems and Applications, 2009. 
[15] A.S. Morse. Supervisory control of families of linear set-point controllers - part 1: Exact matching. IEEE Transactions on Automatic Control, 41:1413-1431, 1996.

[16] P. Peleties and R.A. DeCarlo. Asymptotic stability of $m$-switched systems using Lyapunov-like functions. In American Control Conference, 1991.

[17] A. R. Teel and N. Kapoor. The $\mathcal{L}_{2}$ anti-windup problem: IIts definition and solution. In European Control Conference, 1997.

[18] M.C. Turner and D.J. Walker. Modified linear quadratic bumpless transfer. In American Control Conference, 1999.

[19] M.C. Turner and D.J. Walker. Linear quadratic bumpless transfer. Automatica, 36:1089-1101, 2000.

[20] L. Zaccarian and A. R. Teel. A common framework for anti-windup, bumpless transfer and reliable designs. Automatica, 38(10):17351744, 2002.

[21] L. Zaccarian and A. R. Teel. The $\mathcal{L}_{2}\left(l_{2}\right)$ bumpless transfer problem for linear plants: Its definition and solution. Automatica, 41(7):1273$1280,2005$. 